This item was submitted to Loughborough's Research Repository by the author.

Items in Figshare are protected by copyright, with all rights reserved, unless otherwise indicated.

\title{
Analytical prediction of shear angle and frictional behaviour in vibration- assisted cutting
}

PLEASE CITE THE PUBLISHED VERSION

https://doi.org/10.1016/j.jmapro.2020.12.026

PUBLISHER

Elsevier BV

VERSION

AM (Accepted Manuscript)

PUBLISHER STATEMENT

This paper was accepted for publication in the journal Journal of Manufacturing Processes and the definitive published version is available at https://doi.org/10.1016/j.jmapro.2020.12.026

LICENCE

CC BY-NC-ND 4.0

\section{REPOSITORY RECORD}

Bai, Wei, Anish Roy, Lingxi Guo, Jianfeng Xu, and Vadim Silberschmidt. 2020. "Analytical Prediction of Shear Angle and Frictional Behaviour in Vibration-assisted Cutting”. Loughborough University.

https://hdl.handle.net/2134/13482687.v1. 


\title{
Analytical prediction of shear angle and frictional behaviour in vibration-assisted cutting
}

\author{
Wei Bai ${ }^{\mathrm{a}}$, Anish Roy ${ }^{\mathrm{b}}$, Lingxi Guo ${ }^{\mathrm{c}}$, Jianfeng $\mathrm{Xu}^{\mathrm{a}}{ }^{\mathrm{a} *}$, Vadim V. Silberschmidt ${ }^{\mathrm{b}}$ \\ ${ }^{a}$ State Key Lab of Digital Manufacturing Equipment and Technology, Huazhong University of Science and Technology, Wuhan, 430074, China \\ ${ }^{\mathrm{b}}$ Wolfson School of Mechanical, Electrical and Manufacturing Engineering, Loughborough University, Leicestershire, LE11 3TU, UK \\ ' Shanghai Aerospace Control Technology Institute, Shanghai, 201109, China
}

\begin{abstract}
Vibration-assisted cutting (VAC), a promising technique, proved to enhance the machinability of difficult-to-cut materials. Its significant superiority with regard to conventional cutting (CC) is considered to be closely related to variation of a shear angle in the primary shear zone and specific frictional behaviour at tool-chip interface. This paper analyses kinematics of VAC, focusing on critical stages of tool-workpiece interaction. Based on the evolution of kinematic parameters, a transient shear angle and a tool-chip contact length are investigated in a cycle according to these stages. To predict the transient parameters, an analytical model of the cutting process is proposed based on non-equidistant shear-zone and tool-chip sliding-sticking zone theories. This model for VAC can not only predict the dominant parameters of the cutting process (e.g., cutting force, friction coefficient), but also the secondary ones (e.g., shear strain). Experimental validation of the developed model is performed with orthogonal VAC of titanium alloy, and the shear angles are measured with optical microscopy of chip samples. For various process parameters, the effective shear angle in VAC is larger than that in CC. However, the average shear angle in VAC is smaller than the shear angle in CC. The proposed model can not only effectively predict the shear angle and frictional behaviour in VAC, but also other process parameters in a vibration cycle, enriching the theory of the VAC process.
\end{abstract}

\author{
Keywords: \\ Vibration-assisted cutting \\ Shear angle \\ Tool-chip contact length \\ Non-equidistant shear zone \\ Tool-chip sliding-sticking zone \\ Analytical model
}

\section{Introduction}

Vibration-assisted cutting (VAC) is a promising process, with various benefits compared to conventional cutting (CC). In VAC, micro-scale high-frequency vibration is applied to improve the machinability of difficult-to-machine materials. A wide range of applications demonstrated that it has several advantages such as reduction in the average cutting force, improvement of the cutting stability and surface quality, increase in tool life, etc. [1]. The cutting mechanism of VAC, especially in a vibration cycle, is not clear as a result of the evolution of complex thermo-mechanical processes at high-frequency vibration. For a complex cutting process, two main mechanisms can be identified: first, formation of a chip along the primary shear zone with intense shearing; second, a sustained movement of the chip along the tool-chip interface accompanied by friction effects and formation of a secondary shear zone [2].

In a cutting process, the shear angle is considered as a fundamental parameter that defines plastic deformation and the process geometry
[3]. It is important to have an access to a local distribution of tribological parameters controlling the tool-chip contact [4]. For the plane-strain conditions of orthogonal cutting, once the shear angle and tool-chip friction are defined, all other process parameters could be predicted [3-5].

The shear and frictional behaviours in cutting were studied with three fundamental approaches: empirical, analytical and numerical. A slip-line field was constructed in the chip-formation zone obtained from experiments to predict a shear angle by Oxley [6]. Wright [7] proposed an upper-bound analysis to predict a shear angle using strain-hardening characteristics of a workpiece. Besides, the Lee and Shaffer's [8] and Merchant's [9] shear-angle laws are widely adopted in orthogonal cutting, based on the principles of the maximum shear stress and the minimum energy, respectively. For tool-chip contact mechanics, several approaches were developed to characterize complexity of a tool-chip interface. A broadly used scheme with sticking and sliding zones, located away from a tool tip along its rake face, was proposed to describe the frictional behaviour $[10,11]$. Also, a numerical model can be used to

\footnotetext{
* Corresponding author.

E-mail address: jfxu@hust.edu.cn (J. Xu).
} 


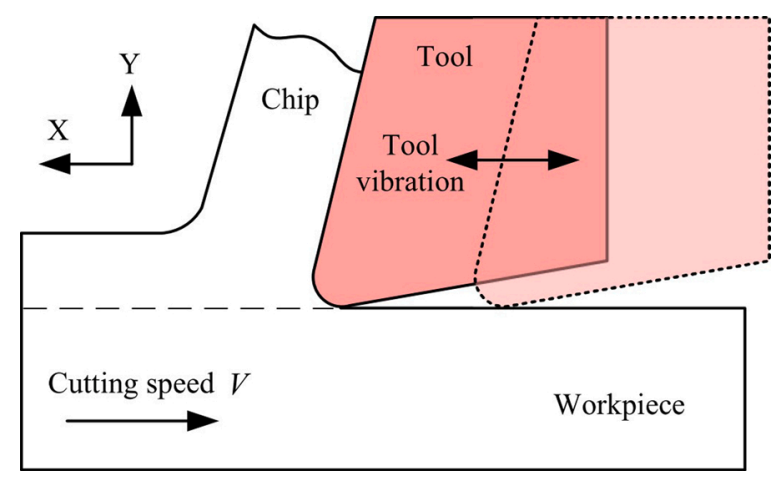

Fig. 1. Schematic diagram of VAC process.

analysis the contact characteristics [12]. Özel [13] employed a thermo-mechanical updated Lagrangian finite-element formulation accounting for the effects of friction of a tool-chip interface friction to simulate the chip formation in orthogonal cutting.

For VAC processes, the reduction of cutting forces was widely reported. Researchers tried to explain the reason of this phenomenon through the increase in a shear angle and the decrease in friction. However, the research on the shear-angle and frictional behaviours in VAC, especially their evolution in a single cycle of vibration, are still rare. Hu and Shao [14] proposed a slip-line cutting model of ultrasonically assisted cutting, that demonstrated that the shear angle in VAC was larger than that in CC. Zhang et al. [15] investigated the instantaneous effective rake and shear angles for different magnitudes of undeformed chip thickness in VAC. They also proposed an analytical model, derived from the force analysis of elastic-plastic deformation steps of low-frequency VAC for an equivalent shear angle [16]. However, they only predicted the equivalent shear angle for VAM. Bai et al. [17] analysed the changes of transient shear angle during a vibration cycle by dividing the cutting process into three stages in elliptical vibration cutting. In addition, some studies investigated the effect of vibration on frictional behaviour. Amini and Kazemiyoun [18] performed quick-stop cutting experiments to measure the chip topography in vibration-assisted turning, and established that ultrasonic vibration could increase the shear angle and decrease the tool-chip contact length. Jamshidi and Nategh [19] proposed a theory for the tool-chip contact length by considering the effect of vibration on the cutting speed. The tool-chip contact length was expressed as the product of a tool-chip contact length in CC and a velocity correlation function. The latter was obtained as exponential function by fitting the experimental data. A two-dimensional FE model was developed by Lotfi and Amini [20] to simulate a frictional behaviour at tool-chip interface in ultrasonically assisted machining. Similarly, the equivalent length of the sticking zone at tool-chip interface was investigated, not the transient variables. Although some works focused on an equivalent or average shear angle and tool-chip contact length in VAC, a further investigation is needed to reveal the evolution of the shear angle and tool-chip friction behaviour in a vibration cycle.

In this study, a model predicting frictional behaviour and evolution of shear angle in VAC is investigated. First, kinematics of VAC is analysed to define the critical instants of tool-workpiece interaction in Section 2. The transient shear angle and tool-chip contact length are defined in a vibration cycle for these instants in Sections 3 and 4, respectively. A model for prediction of process parameters in VAC is proposed in Section 5, that can predict the dominant and secondary parameters of the cutting process. Also, a series of experiments for validation are performed in Section 6. The paper ends with some concluding remarks in Section 7. It should be stated that our previous work [21] is referred to here to support a new independent model in this paper. The study [21] proposed an analytical model of chip formation for precise prediction of orthogonal process in CC, including the scheme for a primary shear zone and a tool-chip interface. This paper analyses the shear angle (in the primary shear zone) and frictional behaviour (at the tool-chip interface) in VAC; their evolution in a single vibration cycle is modelled but the employed relationships between internal parameters
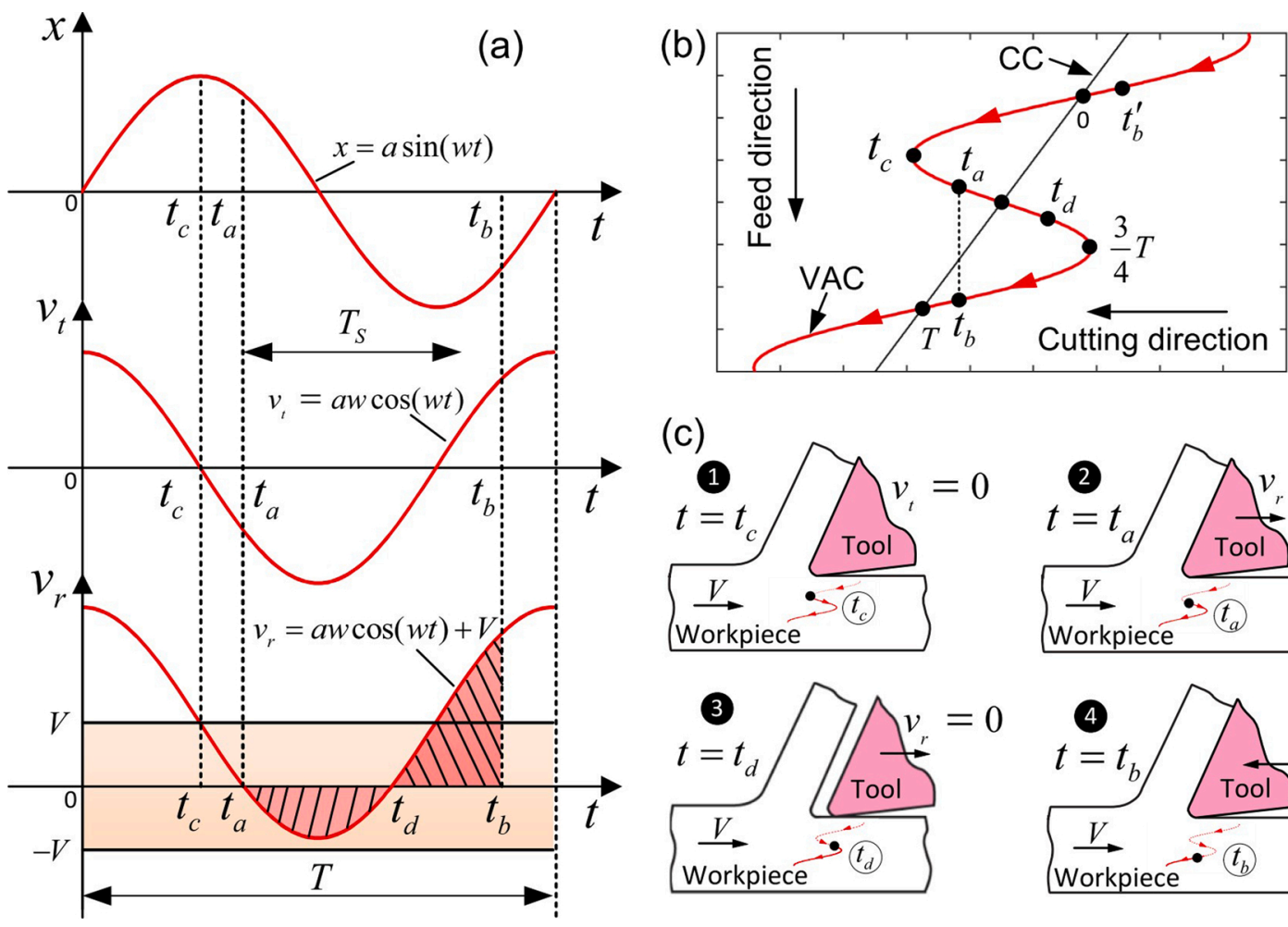

(c)
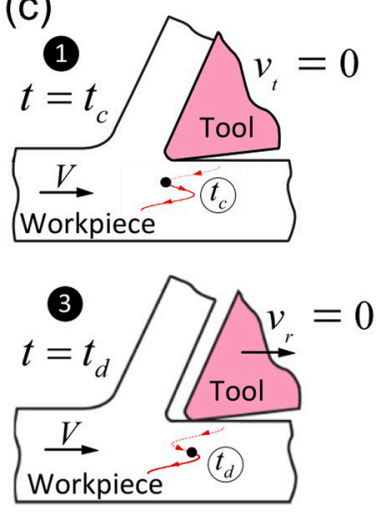
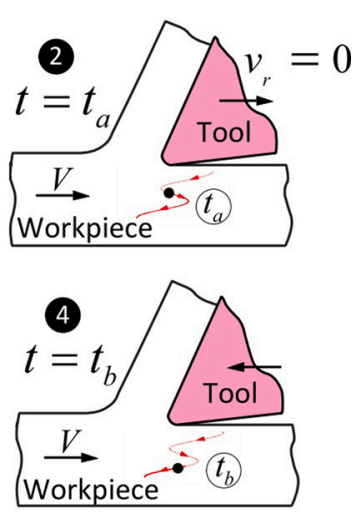

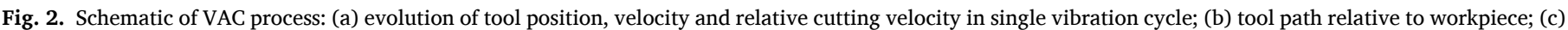
relative positions of tool and workpiece at different times. 
in the primary shear zone and tool-chip interface are obtained from our published model [21].

\section{Kinematics of vibration-assisted cutting process}

During a VAC process, a tool vibrates along the cutting direction at a frequency $f$ (vibration period $T=1 / f$ ). High-frequency vibration of the tool causes the tool to separate periodically from the workpiece as shown in Fig. 1.

The cutting-tool position $x$ in VAC can be presented as follows:

$x=a \sin (\omega t)=a \sin (2 \pi f t)$

where $a$ is the vibration amplitude, $\omega$ is the angular velocity, $t$ is the cutting time.

The tool velocity can be expressed as

$v_{t}=\dot{x}=2 \pi f a \cos (2 \pi f t)$

The relative cutting velocity $v_{r}$ with regard to the workpiece at cutting speed $V$ can be obtained as

$v_{r}=v_{t}-(-V)=2 \pi f a \cos (2 \pi f t)+V$

The graphs of evolution of the tool position $x$, the tool velocity $v_{t}$ and the relative cutting velocity $v_{r}$ with time are shown in Fig. 2(a). The maximum vibration velocity of the tool is $v_{\operatorname{tmax}}=2 \pi f a$. The VAC process should fulfil the condition $v_{\text {tmax }}>V$ for the tool and the workpiece to separate. Therefore, the cutting speed of the separated vibration cutting process should be less than the critical cutting speed $v_{c r}$, namely:

$V<v_{c r}=2 \pi f a$

During the cutting process, the relative cutting velocity is zero $\left(v_{r}=\right.$ 0 ) when the tool disengages from the tool-chip interface, where the disengagement instant is $t_{a}$ (Fig. 2(a)), as follows:

$2 \pi f a \cos \left(2 \pi f t_{a}\right)+V=0$

After the tool and the workpiece are separated, the relative cutting velocity is zero again at the instant $t_{d}$. But the tool and the workpiece keep separation until the tool-workpiece reengagement moment $t_{b}$. The instant $t_{b}^{\prime}$ is the same moment in the last vibration cycle. Thus, the total area of the shaded part in Fig. 2(a) is zero, which can be expressed as

$\int_{t_{a}}^{t_{b}}(2 \pi f a \cos (2 \pi f t)+V) d t=0$

In addition, the tool path relative to the workpiece is shown in Fig. 2 (b), which presents the relationship of the tool-tip trajectory between CC and UAC when the cutting tool moves relative to the workpiece in both cutting and feed directions. Besides, it also shows the important instants $\left(t_{a}, t_{b}, t_{c}, t_{d}\right.$ and $\left.t_{b}^{\prime}\right)$ when the tool-tip moves along the path. Furthermore, the relative positions of the tool and the workpiece at different times are illustrated in Fig. 2(c). At instant $t_{c}$, the tool's velocity reduces to zero. The relative cutting velocity is zero at the instants $t_{a}$ and $t_{d}$.

In each cycle, the separation time $T_{S}$ and the cutting time $T_{C}$ can be expressed as follows:

$T_{S}=t_{b}-t_{a}$

$T_{C}=T+t_{a}-t_{b}$

Then the tool-workpiece contact ratio $r_{t w}$ is obtained as

$r_{t w}=\frac{T_{C}}{T}$

The separation distance travelled by the tool in a single cycle can be calculated by the hatched area from $t_{a}$ to $t_{d}$. However, the effective cutting length is measured for a period from the tool-workpiece reengagement to their next separation. In a single vibration cycle, $D_{c}$ is the

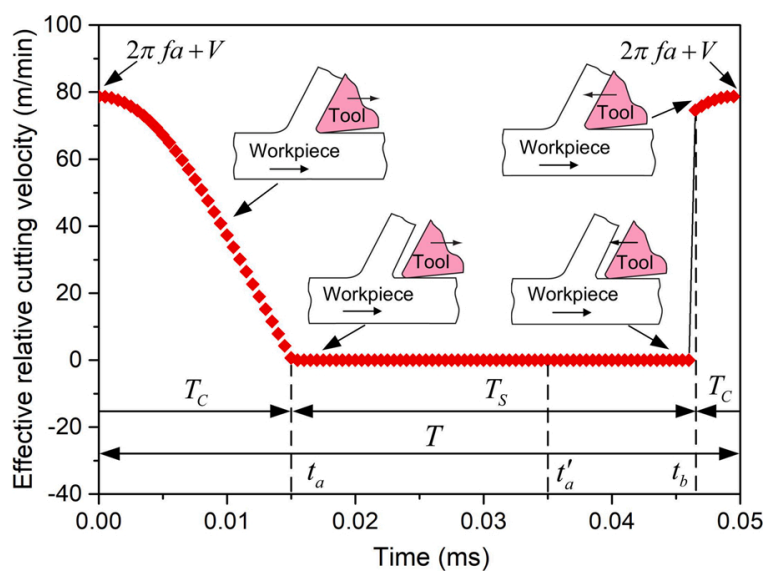

Fig. 3. Evolution of effective relative cutting velocity in single cycle of VAC.

cutting distance and $D_{S}$ is the separation distance between the tool and the workpiece (noncutting distance). They can be calculated from the tool path based on $t_{a}$ and $t_{b}$ (Fig. 2). Therefore, the cutting distance $D_{c}$ and the separation distance $D_{S}$ between the tool and workpiece in each cycle can be calculated as

$D_{C}=\int_{t_{b}}^{T+t_{a}}(2 \pi f a \cos (2 \pi f t)+V) d t=V T$

$D_{S}=-\int_{t_{a}}^{\frac{3}{4} T}(2 \pi f a \cos (2 \pi f t)+V) d t=a \sin \left(2 \pi f t_{a}\right)+V t_{a}+a-\frac{3}{4} V T$

The relevant kinematic parameters of VAC can be calculated from the above formulas, where the instants of tool-workpiece separation $t_{a}$, their reengagement $t_{b}$ and that when the relative cutting velocity is equal to the magnitude of the cutting speed $V\left(t_{c}\right)$ are used below as important moments of time in a cycle of VAC.

Evolution of thermo-mechanical behaviour in a cycle of VAC is complex and the cutting mechanism is not clear. In order to reveal the evolution of physical parameters in the VAC process, several important variables are investigated and the modelling theory of orthogonal cutting is utilized. The effect of material's elasticity on chip separation is not considered in this paper. The shear angle and the tool-chip contact length are two important parameters of the primary and secondary shear zones during the cutting process, analysed for a single cycle of vibration in this paper.

When the relative cutting velocity calculated with Eq. (3) is negative, the tool's rake face is not in contact with the chip, i.e. there is no effective cutting. However, although the relative cutting velocity is positive from the moment $t_{d}$ to $t_{b}$, the tool and the workpiece are still in the separated stage. Therefore, when the tool-workpiece reengagement occurs in the instant $t_{b}$, the cutting starts at a relatively high velocity. The effective relative cutting velocity $v_{e r}$ is defined as the level of relative cutting velocity when the effective cutting happens in a cycle of VAC as

$v_{e r}=\left\{\begin{array}{c}v_{r}, 0 \leq t<t_{a} \\ 0, t_{a} \leq t \leq t_{b} \\ v_{r}, t_{b}<t \leq T\end{array}\right.$

As shown in Fig. 3, the relative cutting velocity begins to decrease at the highest relative cutting velocity $2 \pi f a+V$. At the instant $t_{a}$, when the tool and workpiece are separated, the distance between them gradually decreases until the reengagement instant $t_{b}$, and then the tool continues to cut the workpiece until the relative cutting velocity reaches the maximum again.

In this paper, the effective relative cutting velocity is employed to analyse the tool-chip contact length and the shear angle in a single vibration cycle. A study of the difficult-to-cut material Ti6Al4V is chosen, 


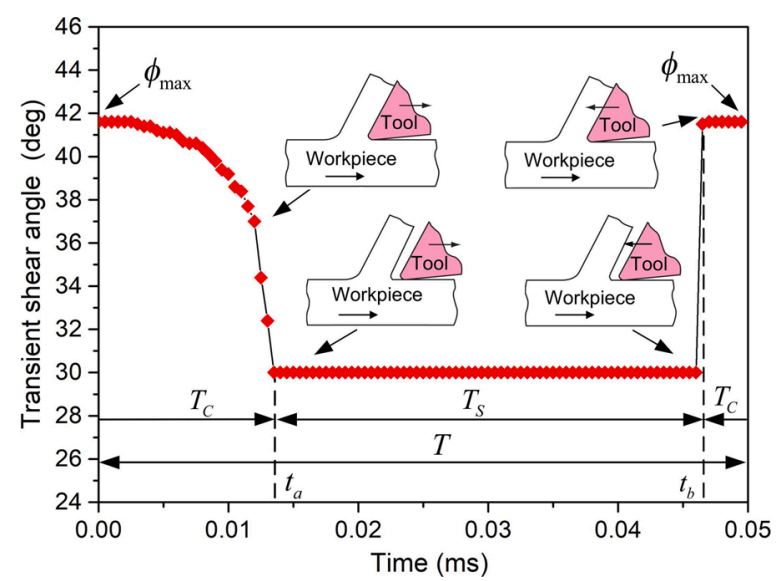

Fig. 4. Evolution of transient shear angle in single cycle of VAC.

with vibration frequency of $20,220 \mathrm{~Hz}$ and amplitude of $7.7 \mu \mathrm{m}$. In the analysis and modelling parts, the cutting speed is $20 \mathrm{~m} / \mathrm{min}$, the feed rate is $0.1 \mathrm{~mm} / \mathrm{rev}$ and the rake angle of the cutting tool is $6.5^{\circ}$; they are used in Sections 3 to 5 . The machining parameters used in the experiments are presented in Section 6.

\section{Shear angle in vibration-asssisted cutting}

As an important parameter of the cutting process, the shear angle affects the shear direction of the primary shear zone and the distribution of forces in the shear zone. The calculation of the shear angle in the VAC process is also divided into several stages.

When the relative cutting velocity reaches its maximum, the shear angle attains the maximum value $\phi_{\max }$. With the reduction of the relative cutting velocity, the shear angle gradually decreases. Similar trends were observed in CC before [21]. The important reason for the decrease of shear angle is that the decrease of relative cutting velocity increases the coefficient of friction, and then raises the friction angle. During this stage, the transient shear angle is $\phi_{t c}$. From $t_{a}$ to $t_{b}$, the tool and workpiece separate and approach each other, but they are not in contact. However, the shear angle reflects a geometric variable of the shear zone. After the tool-workpiece separation, the shear angle is considered as the geometric angle $\phi_{a}$, which is a feature of the separation state. Therefore, the shear angle is calculated for the state when the tool is about to leave the workpiece in the tool-workpiece separation stage. At the instant $t_{b}$, when the tool-workpiece reengages, the shear angle increases instantaneously due to the sharply increased relative cutting velocity, increasing subsequently slowly to the maximum value because of the increase in the relative cutting velocity. Thus, the transient shear angle is expressed as

$\phi_{t}=\left\{\begin{array}{l}\phi_{t c}, 0 \leq t<t_{a} \\ \phi_{a}, t_{a} \leq t<t_{b} \\ \phi_{t c}, t_{b} \leq t \leq T\end{array}\right.$

In the orthogonal cutting process, the shear angle satisfies the following relation:

$\phi=A_{1}+A_{2}(\alpha-\lambda)$

where the material constants $A_{1}$ and $A_{2}$ were identified as $61^{\circ}$ and -1 from experiments [21]. The apparent friction angle $\lambda$ can be obtained as $\tan ^{-1} \mu_{a}$, which is discussed in Section 4.

The transient shear angle $\phi_{t c}$ in a vibration cycle can be calculated through above model as shown in Fig. 4.

\section{Tool-chip frictional behaviour in vibration-asssisted cutting}

At the tool-chip interface, the tool-chip frictional behaviour is also very important for analysis of the VAC process, including the coefficient of friction and the tool-chip contact length. Generally, the tool-chip interface is divided into sticking and sliding zones. The coefficient of friction of the whole contact zone is called as the apparent (global) coefficient of friction, and that of the sliding zone is termed the sliding coefficient of friction.

The relationship between the apparent coefficient of friction $\mu_{a}$ and the sliding coefficient of friction $\mu_{s l}$ at the tool-chip interface was derived by Bai et al. [21] as

$\mu_{s l}=\frac{\tau_{s y}}{P_{0}} \frac{1}{\left[1-\frac{1}{\xi}\left(\frac{\mu_{a} P_{0}}{\tau_{s y}}-1\right)\right]^{\xi}}$

where the yield shear stress of the material $\tau_{s y}$ is equal to the shear stress at the exit of the primary shear zone. And the pressure on the tool tip $P_{0}$ can also be calculated by the shear stress at the exit of the primary shear zone. The distribution of shear stress in the primary shear zone is evaluated through the non-equidistant shear zone model reported in [21]. The pressure-distribution exponent $\xi$ is assumed as 2 according to Childs et al. [22]. In our proposed model, the apparent coefficient of friction $\mu_{a}$ and the sliding coefficient of friction $\mu_{s l}$ are finally determined by several iterations for a given initial value of $\mu_{a}$ in Section 5 .

The tool-chip contact length is the key variable for the tool-chip interface. It defines the length of the interaction between the tool's rake face and the workpiece and the zone of heat exchange in the rake face, which can be utilized to determine other parameters in the secondary shear zone.

In VAC, the tool-chip contact length changes with the periodic separation of the tool's rake face and the chip. Therefore, this section analyses the transient tool-chip contact length in VAC. In a single vibration cycle of VAC, the tool has no contact with the chip at the stage when the effective relative cutting velocity is zero; the transient tool-chip contact length is also zero. When the relative cutting velocity is larger than the cutting speed, the transient tool-chip contact length $L_{t c}$ can be calculated with the conventional theory of orthogonal cutting. When the relative cutting velocity is equal to the cutting speed, the tool-chip contact length at this instant is considered to be the unique value $L_{c c}$ determined by the cutting speed. The tool-chip contact length is assumed to be positively related to the relative cutting velocity because of the tool is about to separate from the workpiece. Thus, the transient tool-chip contact length in a cycle can be obtained as

$L_{c t}=\left\{\begin{array}{c}L_{t c}, v_{r}>V \\ L_{c c}, v_{r}=V \\ L_{c c} \frac{v_{r}}{V}, 0<v_{r}<V \\ 0, v_{r}=0\end{array}\right.$

The transient tool-chip contact length is piecewise modelled according to the nominal cutting velocity $V$ because the cutting state changes at velocities above or below it. The essential reason is that the condition when the relative cutting velocity equals to the nominal cutting velocity $\left(v_{r}=V\right)$ is critical for the start of separation between the tool and the workpiece. To further clarify the characteristics of this piecewise function, the expression of the transient tool-chip contact length can be rewritten for time interval as follows:

$L_{c t}=\left\{\begin{array}{c}L_{t c}, 0 \leq t<t_{c} \\ L_{c c} \frac{v_{r}}{V}, t_{c} \leq t<t_{a} \\ 0, t_{a} \leq t<t_{b} \\ L_{t c}, t_{b} \leq t \leq T\end{array}\right.$

According to the modelling theory for the secondary deformation 


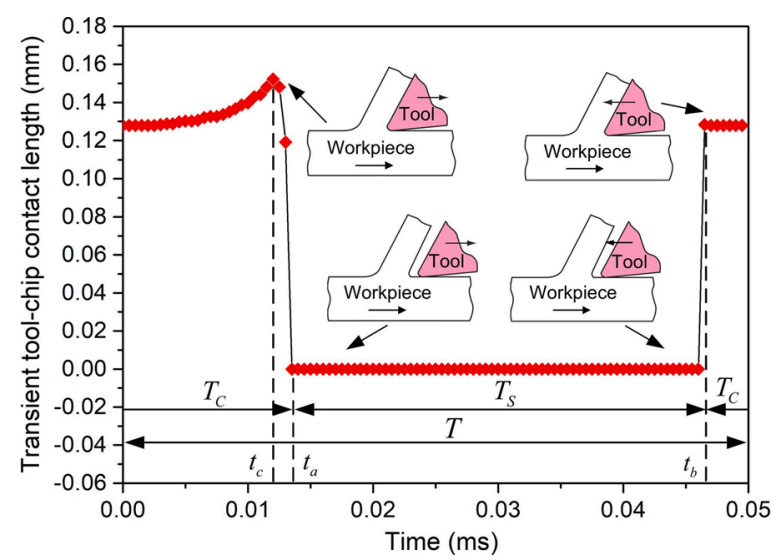

Fig. 5. Evolution of transient tool-chip contact length in single cycle of VAC.

zone in an orthogonal cutting process [21], the tool-chip contact length can be calculated as

$L_{C}=t_{u} \frac{\sin (\varnothing+\lambda-\alpha)}{\sin \phi \cos \lambda}\left(\frac{2}{3}+\frac{1+2(\pi / 4-\phi)}{3 \tan (\phi+\lambda-\alpha)}\right)$

where $t_{u}$ is the undeformed chip thickness. $\phi, \lambda$ and $\alpha$ are the shear angle, apparent friction angle of the tool-chip interface and the tool rake angle, respectively. When the relative cutting velocity is greater than or equal to the cutting speed, the tool-chip contact lengths $L_{t c}$ and $L_{c c}$ can be obtained from Eq. (18) as the values of tool-chip contact length $L_{C}$ at different instants.

The transient tool-chip contact length in a vibration cycle calculated with Eq. (17) is presented in Fig. 5. Since the relative cutting velocity gradually decreases from $t=0$ to the instant $t_{c}$, the tool-chip contact length increases slightly, as also concluded by Bai et al. [21]. The main reason for this is that the decrease of the relative cutting velocity leads to slowly growing tool-chip friction and then increases the friction angle, resulting in a slight increase in the transient tool-chip contact length. Subsequently, due to the further reduction of the relative cutting velocity, the tool and the workpiece are about to separate. This unsteady process is different from the previous stage, where the tool-chip contact length decreases sharply due to the physical separation. From $t_{a}$ to $t_{b}$, the tool and workpiece are completely separated, and the tool-chip contact length is zero. After the instant $t_{b}$, the tool reengages with the workpiece, and the contact length slowly decreases as the higher relative cutting velocity continues to increase. The reason here is opposite to that in the cutting process from $t=0$ to $t_{c}$. At the instant, the transient tool-chip contact length reaches the minimum in the cutting stage (from $t_{b}$ to $T+t_{a}$ ) due to the peak of effective relative cutting velocity (Fig. 3). After that moment $(T)$, the cutting process enters the next vibration cycle and the transient tool-chip contact length starts to increase as the relative cutting velocity decreases from $T$ to the instant $T+t_{c}$.

\section{Analytical modelling and parameter prediction}

At present, there is no established theory for unsteady-state cutting. Scholars usually use the finite-element method or adopt the steady-state cutting theory but consider the main factors to modify the model. This paper selected the latter. The evolution of important variables in UAC of Ti6Al4V presented using the finite-element method in Bai et al. [23] demonstrated that the strain, strain rate and temperature fluctuated with the tool vibration. Thus, there is reason to believe that these variables in the VAC process change at high frequency. In addition, the steady-state cutting theory used in this paper is mainly the non-equidistant shear zone model, and the unsteady process affects the deflection of the primary shear band significantly. In fact, the experimental results below show that the shear angle changes by about $4 \sim 6^{\circ}$ in VAC. Thus, the deflection angle is very small compared to the size of a long narrow shear band. It is considered that the unsteady-state process has little influence on the use of the shear-zone theory. Therefore, the chip-formation theory of orthogonal cutting is adopted in this paper.

Based on the above analysis of the transient tool-chip contact length and the transient shear angle, the VAC process is modelled by combining it with the chip-formation theory of orthogonal cutting. The flow chart for calculation of the process parameters for VAC is shown in Fig. 6. The non-equidistant shear-zone model is adopted for the primary shear zone, where a straight band of thickness $h$ is divided in proportion $k$. The main shear plane $A B$, the inlet shear plane $C D$ and the outlet shear plane $E F$ are parallel. Two coordinate systems are defined: $\Re=\{0, x, y, z\}$ and $\Re_{s}=\left\{\mathbf{o}, x_{s}, y_{s}, z_{s}\right\}$ - for the tool-workpiece movement system and the material shear flow system. This model can be used to obtain the levels of strain, strain rate, temperature and shear stress in the primary shear zone. They are transfered to the secondary shear zone (SSZ) in order to calculate the chip thickness and pressure on the tool tip. Then, a sticking-sliding zone model is utilized at the tool-chip interface to

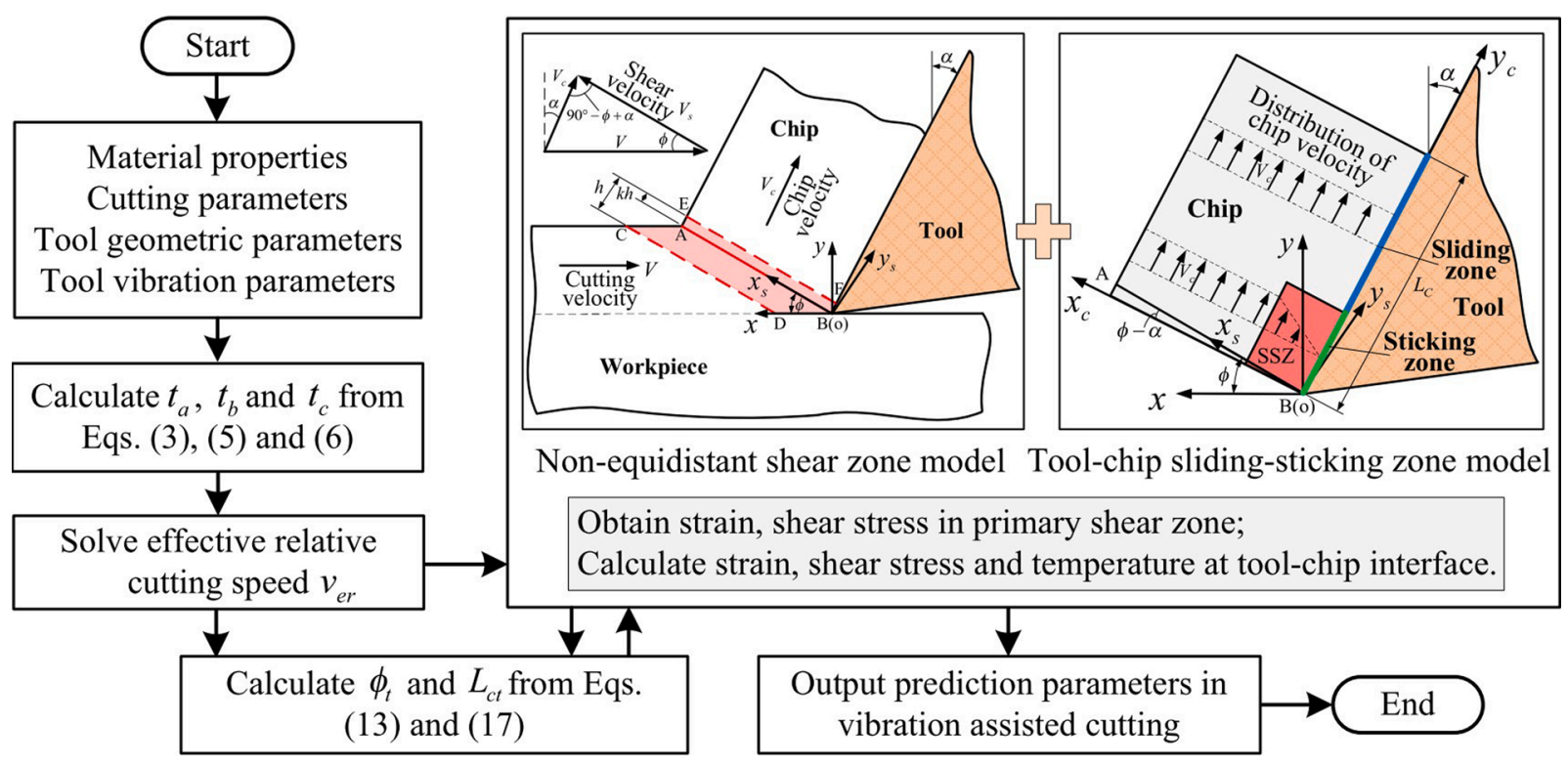

Fig. 6. Flow chart for calculation of process parameters for VAC. 

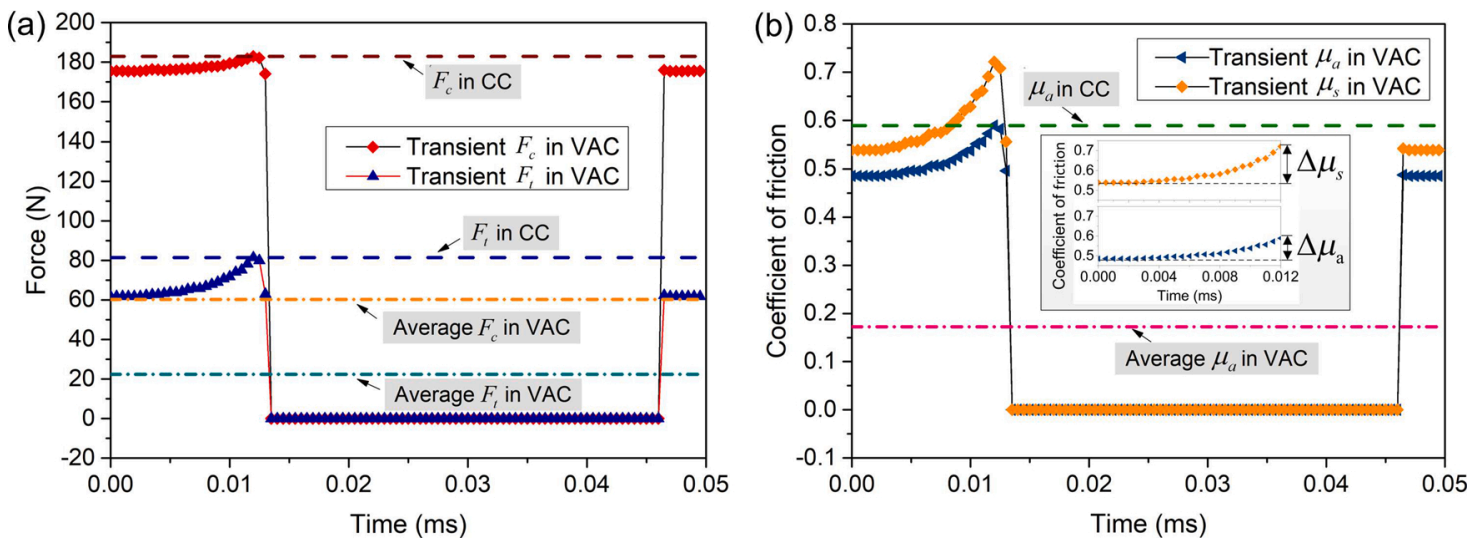

Fig. 7. Evolution of cutting forces (a) and coefficient of friction (b) in single cycle of VAC.

predict the apparent and sliding coefficients of friction, the tool-chip contact length and the length of the sticking zone, the equivalent strain together with average temperature. Another coordinate system $\Re_{c}=\left\{\mathbf{o}, x_{c}, y_{c}, z_{c}\right\}$ is defined for the chip flow system. More details are available in our previous work [21]. Thus, the transient shear angle and the tool-chip contact length can be determined. The shear angle $\phi$ is selected, when the shear stress at the tool-chip interface $\tau_{\text {int }}$ (calculated based on the resultant force at the primary shear zone) equals the shear flow stress at the tool-chip interface $k_{\text {int }}$ (corresponds to the strain, strain rate and temperature at the tool-chip interface). The transient shear angle $\phi_{t c}$ and $\phi_{a}$ are the values of shear angle $\phi$ at different times. This analytical model for the VAC process can be used to predict the dominant parameters such as cutting force, friction coefficient, etc., as well as the secondary parameters such as shear strain, etc.

Thus, the cutting force $\left(F_{c}\right)$ and the thrust force $\left(F_{t}\right)$ can be expressed as

$F_{c}=\left\{\begin{array}{c}\frac{w t_{u} \tau_{A B} \cos (\lambda-\alpha)}{\sin \left(\phi_{t c}\right) \cos \left(\phi_{t c}+\lambda-\alpha\right)}, 0 \leq t<t_{a} \\ 0, t_{a} \leq t<t_{b} \\ \frac{w t_{u} \tau_{A B} \cos (\lambda-\alpha)}{\sin \left(\phi_{t c}\right) \cos \left(\phi_{t c}+\lambda-\alpha\right)}, t_{b} \leq t \leq T\end{array}\right.$
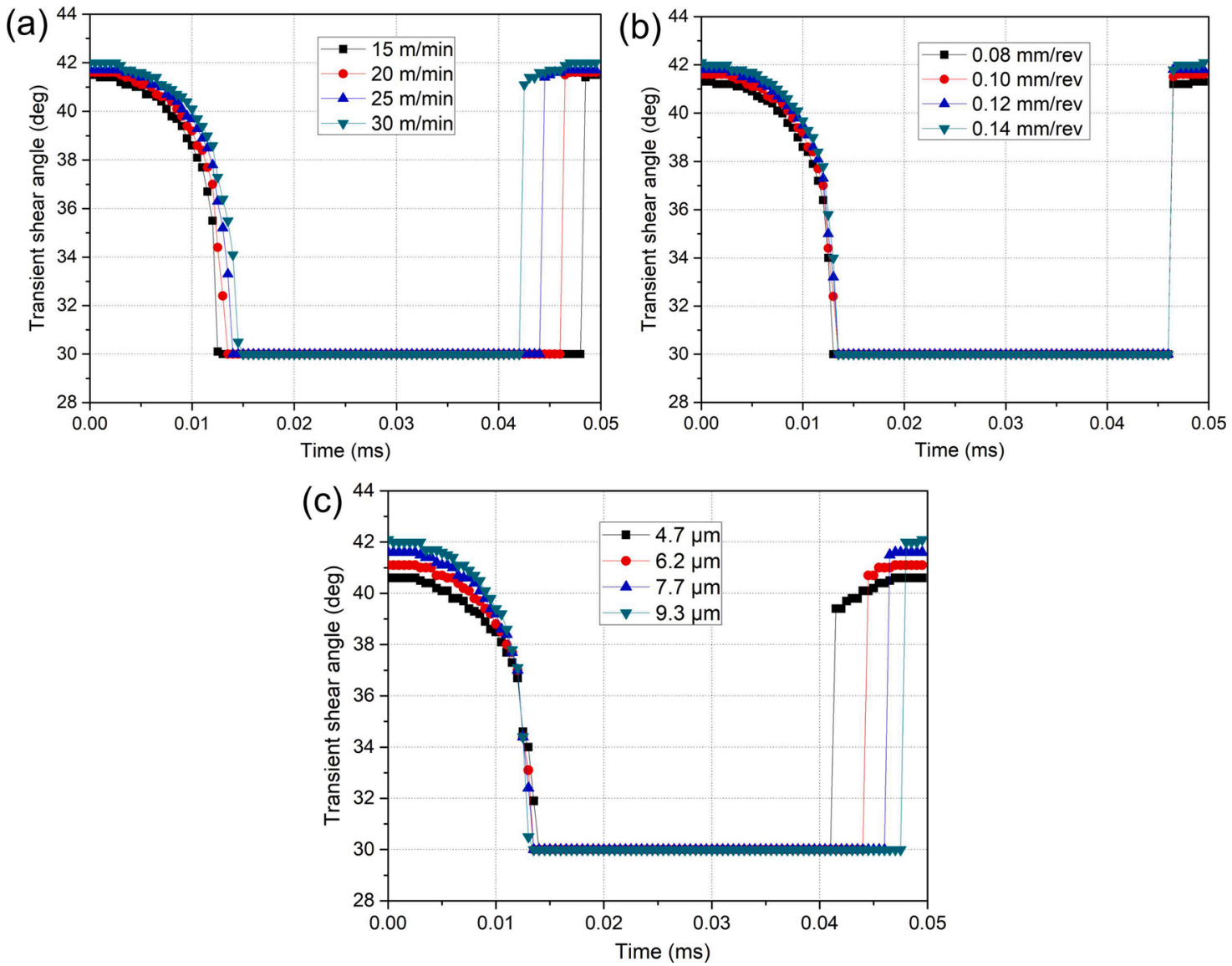

Fig. 8. Effect of processing parameters on transient shear angle in single cycle of VAC: (a) cutting speed; (b) feed rate; (c) vibration amplitude 

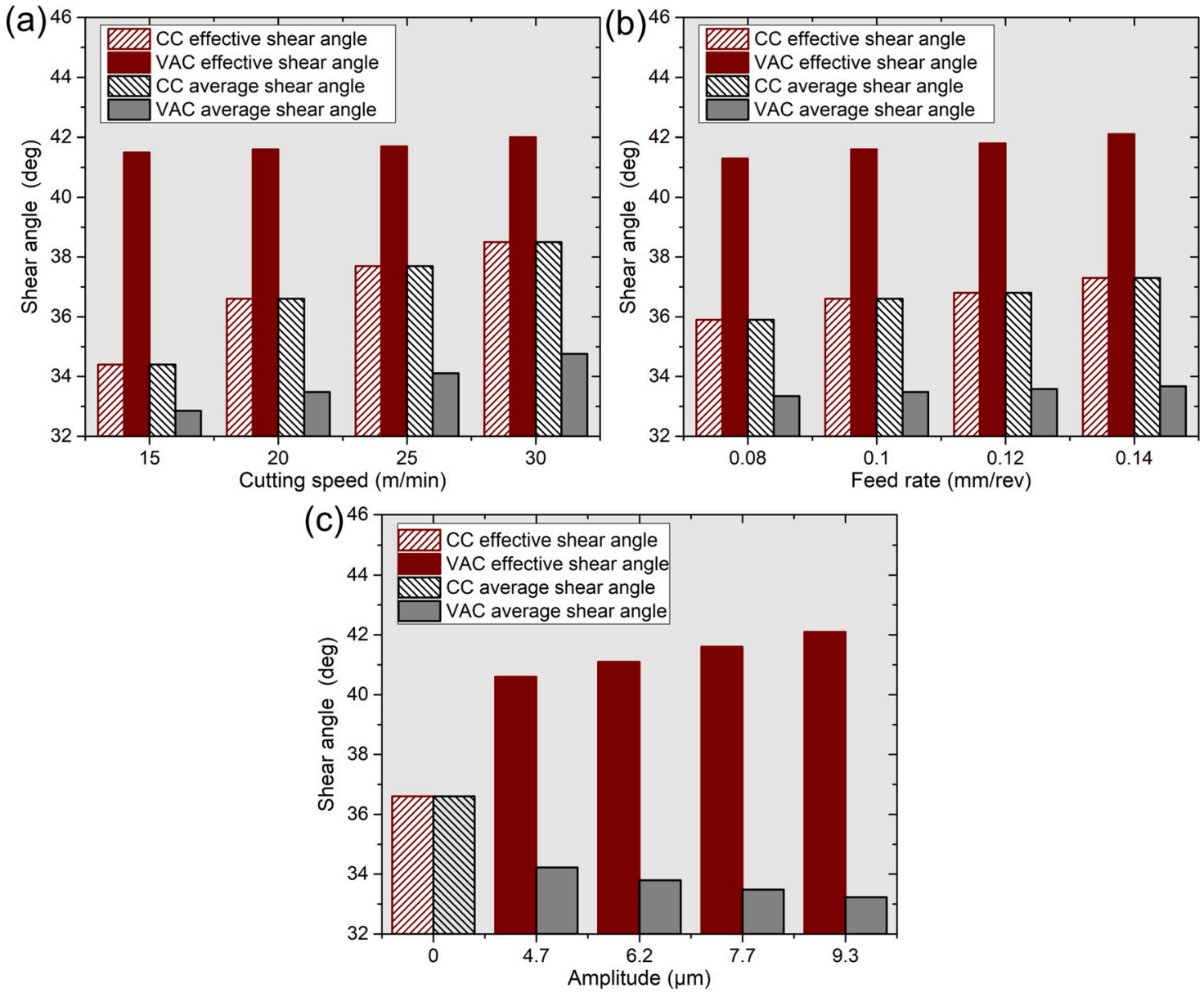

Fig. 9. Comparison of shear angle in CC and VAC for various process parameters: (a) cutting speed; (b) feed rate; (c) vibration amplitude.

$F_{t}=\left\{\begin{array}{c}\frac{w t_{u} \tau_{A B} \sin (\lambda-\alpha)}{\sin \left(\phi_{t c}\right) \cos \left(\phi_{t c}+\lambda-\alpha\right)}, 0 \leq t<t_{a} \\ 0, t_{a} \leq t<t_{b} \\ \frac{w t_{u} \tau_{A B} \sin (\lambda-\alpha)}{\sin \left(\phi_{t c}\right) \cos \left(\phi_{t c}+\lambda-\alpha\right)}, t_{b} \leq t \leq T\end{array}\right.$

where the cutting width $w$ is $1 \mathrm{~mm}$, the undeformed chip thickness $t_{u}$ is numerically equal to the feed rate. The shear stress $\tau_{A B}$ at the primary shear plane $\mathrm{AB}$ (Fig. 6) is obtained from the non-equidistant shear-zone model [21].

The evolution of cutting force and the tool-chip coefficient of friction in a single cycle of VAC is shown in Fig. 7.

As seen from the force curve (Fig. 7(a)), the main cutting $\left(F_{c}\right)$ and thrust $\left(F_{t}\right)$ forces are zero in the stage of separation of the tool and the workpiece. At the beginning of the vibration cycle (from 0 to $t_{c}$ ), the cutting force increases slowly due to the decrease of the relative cutting velocity, which is consistent with the results reported for CC [21]. From $t_{c}$ to $t_{a}$, the cutting forces decrease sharply due to the separation of the tool and the workpiece, and then decrease slowly because of the increase of the relative cutting velocity after the instant $t_{b}$. In addition, the tool-workpiece contact ratio $r_{t w} \ll 1$; thus, many researchers concluded that the average cutting force in VAC is lower than that of CC. Actually, comparison of the main cutting and thrust forces in CC with the average cutting forces in VAC shows clearly that the VAC reduces the average cutting forces significantly.

The changed trends of apparent $\left(\mu_{a}\right)$ and sliding $\left(\mu_{s}\right)$ coefficients of friction (Fig. 7(b)) are similar to that of cutting forces, but the growth rate of the sliding coefficient of friction $\left(\Delta \mu_{s}\right)$ is more significant than that of the apparent one $\left(\Delta \mu_{a}\right)$ at the stage when the relative cutting velocity reduces. The main reason is that the sticking zone near the tool tip at the tool-chip interface is not sensitive to the cutting speed; thus, the apparent (global) coefficient of friction is less affected by the variation of the relative cutting velocity than the sliding coefficient of friction.

Besides, the sliding coefficient of friction is always slightly higher than the apparent one, corresponding to the reported results [21]. It also can be found that the average coefficient of friction is less than the coefficient of friction in CC.

However, the parameters of VAC are very difficult to measure in a single period of vibration as its vibration frequency is normally very high. Therefore, the important parameters - shear angle in VAC — is analyzed and validated in the next section.

\section{Prediction and validation of shear angle in vibration-assisted cutting}

\subsection{Prediction of shear angle for various process parameters}

The transient shear angles are predicted for various processing parameters based on the developed model. The predictions were implemented for the cutting speed of $15 \mathrm{~m} / \mathrm{min}, 20 \mathrm{~m} / \mathrm{min}, 25 \mathrm{~m} / \mathrm{min}, 30 \mathrm{~m} /$ $\min$ and the feed rate of $0.08 \mathrm{~mm} / \mathrm{rev}, 0.10 \mathrm{~mm} / \mathrm{rev}, 0.12 \mathrm{~mm} / \mathrm{rev}$, $0.14 \mathrm{~mm} / \mathrm{rev}$ for vibration amplitude of $4.7 \mu \mathrm{m}, 6.2 \mu \mathrm{m}, 7.7 \mu \mathrm{m}, 9.3 \mu \mathrm{m}$; the vibration frequency was $20,220 \mathrm{~Hz}$. The effect of different processing parameters on the transient shear angle in a single cycle of VAC is shown in Fig. 8. Overall, the trends for the shear angle under various machining parameters are similar, with the main change caused by the 

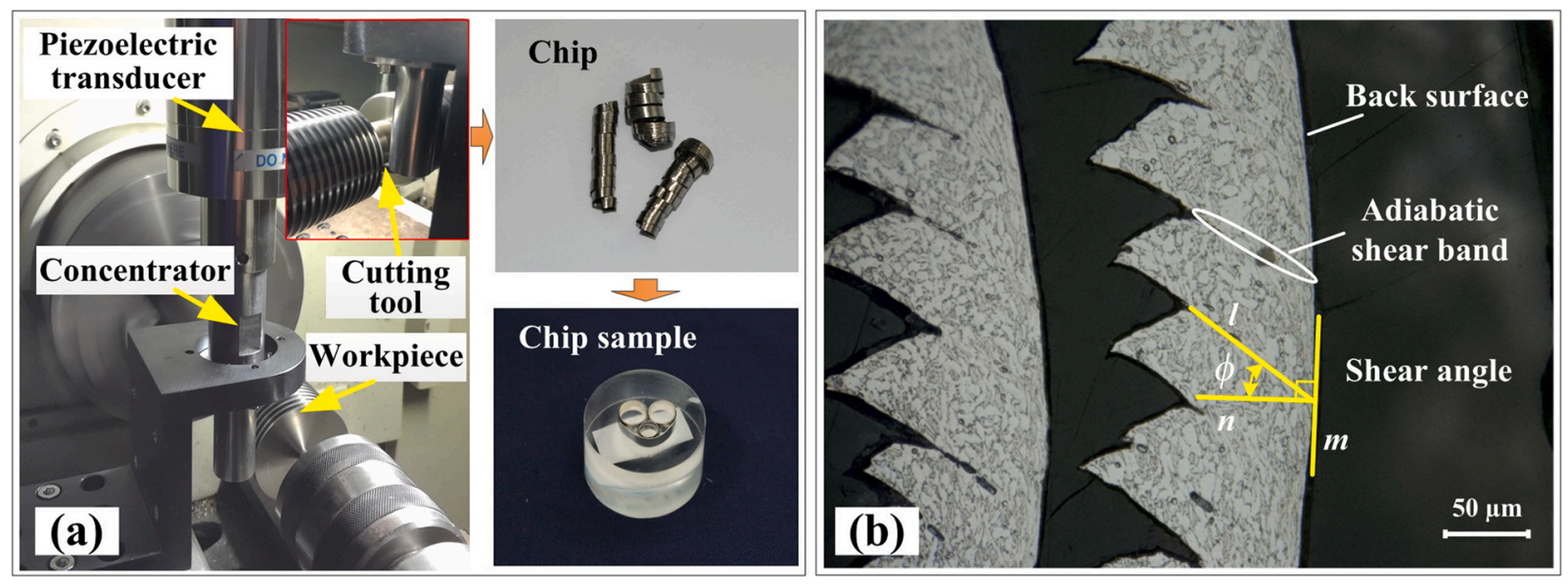

Fig. 10. Experimental work: (a) sample preparation; (b) measurement of shear angle.

separation of tool from the workpiece in the cycle. For different cutting speeds, the transient shear angle increase slightly with the increase in the speed in the tool-workpiece cutting stage. Meanwhile, the toolworkpiece separation time was shortened (i.e. the tool-workpiece contact ratio $r_{t w}$ increased) as the cutting speed increased (Fig. 8(a)). For different feed rates, the increase in the rate also caused a slight increase in the shear angle at the cutting stage, but the feed rate did not affect the separation time between the tool and the workpiece (Fig. 8(b)). The increase in the amplitude also improved the transient shear angle at the cutting stage. Besides, the effect of the amplitude on the absolute value of shear angle was greater than that of the cutting speed and the feed rate, mainly because the variation of amplitude had a more significant effect on the relative cutting velocity. In addition, the tool-workpiece separation time was extended (i.e. the tool-workpiece contact ratio $r_{t w}$ decreased) as the amplitude increased (Fig. 8(c)).

In the VAC process, the shear angle always changes in each cycle. However, the level of the maximum shear angle is considered to retain and transfer to the chip as a result of material's flow and rapid cooling of the chip. In this paper, the effective shear angle in a cycle of VAC was defined as the maximum shear angle for prediction. The average shear angle can be calculated from the mean transient shear angle in a cycle. Here, VAC is the special process - ultrasonically assisted cutting because the selected frequency exceeds $20 \mathrm{kHz}$. The prediction of effective shear angle and average shear angle with various process parameters are shown in Fig. 9. Obviously, the average shear angle in CC is equal to the effective shear angle because the shear angle is regarded constant during the cutting process. In general, for different machining parameters, the effective shear angle of VAC is larger than that of CC, while the average shear angle of VAC is smaller than the average shear angle (effective shear angle) of CC. The main reason for this is the fact that the maximum relative cutting speed in VAC is higher than the cutting speed in CC, leading to a larger effective shear angle in VAC. Also, the tool-workpiece separation during the VAC process results in a smaller average shear angle than that in CC.

With the increase in the cutting speed (Fig. 9(a)), the effective shear angle in VAC slightly increases, and it gradually increases in CC. There are several reasons for this. First, the contribution of the cutting-speed improvement compared to the relative cutting speed in VAC is less than that in CC. Second, our previous results [21] indicate that the shear angle increases with an increase in the cutting speed and it is obvious that the shear angle changes more in the low-speed region (cutting speed in CC) than in the high-speed region (relative cutting speed in VAC). In addition, Fig. 9(a) also demonstrates that the average shear angle gradually increases in VAC. Actually, the growing cutting speed leads to the increases in the duty ratio and the effective relative cutting velocity in vibration-assisted cutting, which results in the increase of the average shear angle. With the increase in the feed rate (Fig. 9 (b)), the effective shear angle in both VAC and CC and the average shear angle in VAC increase slightly. In Fig. 9(c), the amplitude of zero corresponds to the CC process. When the vibration amplitude increases in VAC, the effective shear angle grows gradually while the average shear angle decreases. This is a result of a more significant effect of the increase of amplitude on the increase of the relative cutting speed, so the effective shear angle increases. The increase of amplitude also increases the separation time of the tool and the workpiece; thus, the average shear angle in VAC decreases.

\subsection{Experimental work and validation of shear angle}

The parameters of VAC, such as transient tool-chip contact length and transient shear angle, currently cannot be measured in a single period of vibration as vibration frequency in VAC is normally very high. However, the proposed model indicates that these transient parameters are interrelated and ultimately reflected in material's removal in shear and chip formation, and the effective shear angle is the most representative variable that can be measured. Therefore, the experimental validations of shear angle in VAC are performed.

In order to validate the predicted levels of the shear angle, the orthogonal cutting experiment with VAC was performed. The difficultto-cut material Ti6Al4V was selected and the process parameters were the same as in the developed model. As shown in Fig. 10, the ultrasonically assisted cutting device was fixed on the lathe, consisting of the piezoelectric transducer and the concentrator. A cemented-carbide cutting tool was attached to the bottom of concentrator. The workpiece was machined with equidistant grooves, with the cutting edge was parallel to the cylindrical surface of the workpiece so that the orthogonal cutting process was performed corresponding to the 2D plane state. The tool feed is along the radial direction of the workpiece. The obtained chips were spiral columnar, helpful for sample preparation (Fig. 10(a)) and observation. The obtained chips were grinded and polished after hot inlaying, then observed under the metallographic microscope after etching. The shear angle was measured (Fig. 10(b)) for each group of chip samples. In the experiments, a chip-segmentation frequency can be evaluated as $2 \sim 4 \mathrm{kHz}$ for the used cutting speed $(15 \sim 30 \mathrm{~m} / \mathrm{min}$ ) [21]. That is, the vibration frequency $(\sim 20 \mathrm{kHz})$ is much larger than the chip-segmentation frequency. Thus, it is assumed that the variation of shear angle in a vibration cycle does not interfere with the formation of adiabatic shear bands. Therefore, the effective shear angle in VAC can be related to the serrated chip morphology instead of the shear angle at random moment. To measure the shear angle (Fig. 10(b)), it is necessary to draw a straight line $(l)$ along the adiabatic shear band, and draw a tangent $(m)$ passing through the intersection of this line and the back surface of the chip, then build a line ( $n$ ) perpendicular to the tangent line through the intersection. Thus, the shear angle is determined by the 

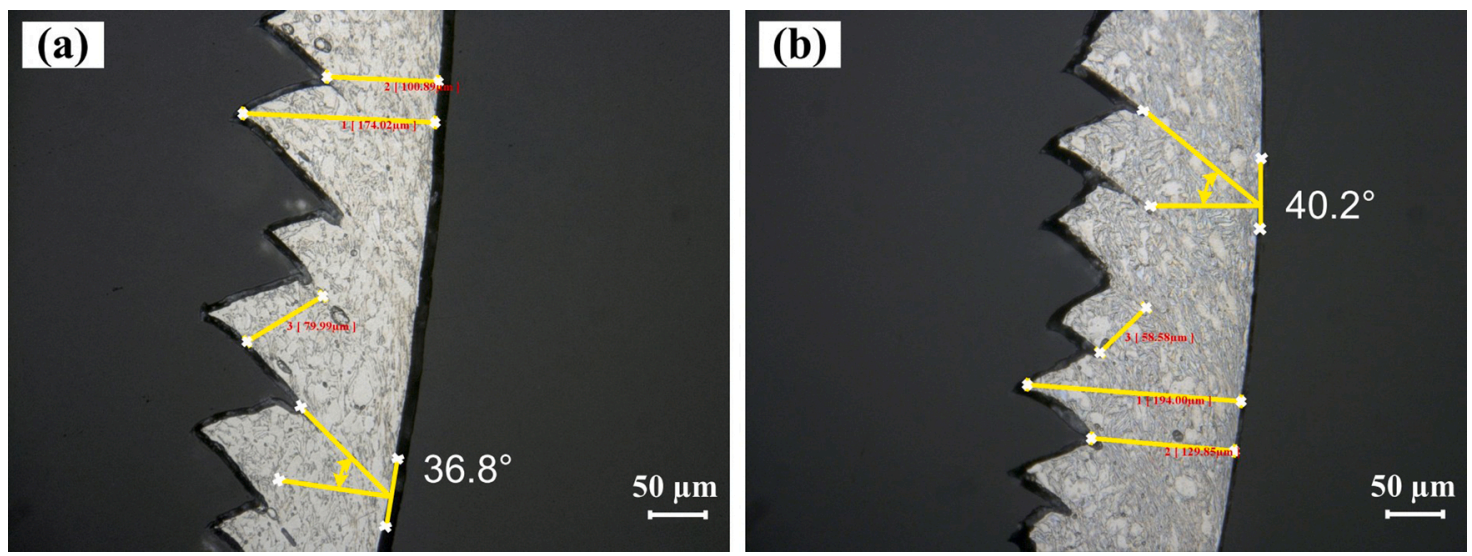

Fig. 11. Typical comparison of chip morphology and shear angle in CC (a) and VAC (b).
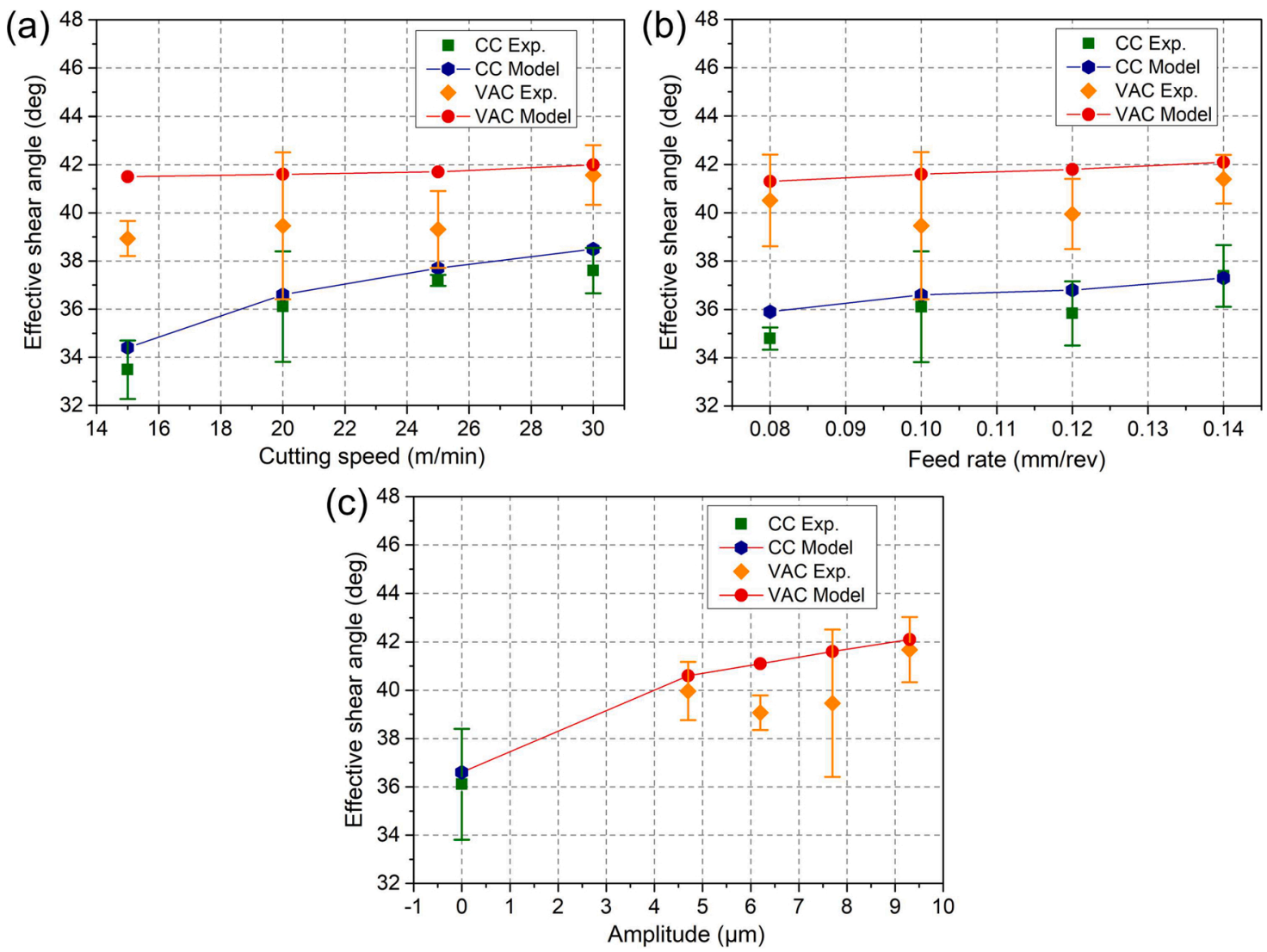

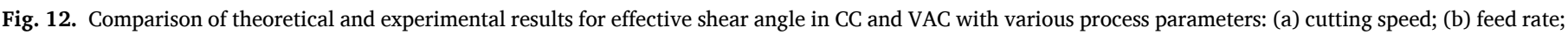
(c) vibration amplitude.

angle between the vertical line $(n)$ and the line passing through the shear band $(l)$.

The experiments were performed for the cutting speed of $15 \mathrm{~m} / \mathrm{min}$, $20 \mathrm{~m} / \mathrm{min}, 25 \mathrm{~m} / \mathrm{min}, 30 \mathrm{~m} / \mathrm{min}$ and the feed rate of $0.08 \mathrm{~mm} / \mathrm{rev}$, $0.10 \mathrm{~mm} / \mathrm{rev}, 0.12 \mathrm{~mm} / \mathrm{rev}, 0.14 \mathrm{~mm} / \mathrm{rev}$ with vibration amplitude of $4.7 \mu \mathrm{m}, 6.2 \mu \mathrm{m}, 7.7 \mu \mathrm{m}, 9.3 \mu \mathrm{m}$; the vibration frequency was $20,220 \mathrm{~Hz}$. The effective shear angles in CC and VAC were obtained based on the above measuring method. A typical comparison of chip morphology and shear angle in CC and VAC is shown in Fig. 11. The cutting speed and the feed rate were set as $20 \mathrm{~m} / \mathrm{min}$ and $0.14 \mathrm{~mm} / \mathrm{rev}$, respectively. A vibration amplitude was $7.7 \mu \mathrm{m}$ for VAC. Apparently, the use of ultrasonic vibration in the cutting process improved the effective shear angle. In addition, the size of sawtooth chip and the number of serrated chips also show some differences. It seems that VAC improves the chip segmentation.

The effective shear angles for CC and VAC were measured in the experiments for different process parameters as shown in Fig. 12. The effective shear angle in a cycle of VAC was defined as the retained shear angle in the chip from the shear-angle graph. The error bar reflects the scatter in measurements of the shear angle at three different chip locations on the same sample. Apparently, the effective shear angle in VAC for different process parameters was indeed higher than that in CC. For different cutting speeds, the effective shear angle in CC increased gradually (Fig. 12(a)). The effective shear angle of in VAC increased slowly with the cutting speed. For various feed rates, the effective shear angle in both VAC and CC increased slowly (Fig. 12(b)). The effective 
shear angle of VAC grows significantly as the vibration amplitude increased (Fig. 12(c)). Overall, the theoretical results are in good agreement with the experiments. However, better matching of the theoretical and experimental results was achieved for CC, while the theoretical values of VAC are slightly higher than the experimental values. The reason could be that final shear angle of chip in the VAC process is not exactly the maximum value, and some shear angles less than the maximum value were retained under some conditions.

\section{Conclusions}

In this paper, the transient shear angle and the tool-chip contact length of one-dimensional VAC were analysed, and the process parameters in VAC were predicted based on the orthogonal cutting theory. In addition, the results predicted for the shear angle were validated with the orthogonal cutting experiment in both CC and VAC. Conclusions from this study can be drawn as follows:

(a) The evolution of the transient shear angle in a vibration cycle can be divided into three stages. The instantaneous shear angle reaches its maximum value when the relative cutting velocity of the tool and workpiece attains the maximum. From the instant that the tool and workpiece separate to the time of their reengagement, the transient shear angle retains the stable value.

(b) The development of the transient tool-chip contact length in a single vibration cycle can be divided into four stages. When the tool velocity is zero, the transient tool-chip contact length reaches the maximum value. The transient tool-chip contact length vanished during the period from the disengagement to the reengagement of the tool and the workpiece. The variation of the transient tool-chip contact length is small from the instant of toolworkpiece reengagement to the instant when the tool velocity reaches zero in the next cycle.

(c) For various process parameters, the effective shear angle in VAC is larger than that in CC. However, the average shear angle in VAC is smaller than the average shear angle (effective shear angle) in CC. The increase of vibration amplitude improves the transient shear angle. In addition, the effect of vibration amplitude on shear angle is more significant than that of cutting speed and feed rate.

(d) The theoretical results for the effective shear angle in CC and VAC are in good agreement with the data of orthogonal cutting experiments.

In this paper, the analysis of the transient shear angle and friction behaviour of VAC can provide the theoretical support for further understanding of the mechanism of VAC and the thermo-mechanical modelling of VAC process. In addition, this paper presents a method and a model with potential to analyze the transient fluctuations of variables in a cycle of VAC that can be used not only for one-dimensional but also multi-dimension vibration-assisted cutting. Although more factors should be considered in multi-dimensional vibration-assisted cutting, the proposed model can provide a reference for further investigations of the mechanisms, underpinning fluctuations of variables. Furthermore, the effectiveness of the model was validated by taking titanium alloy as an example in this paper. However, the proposed approach is applicable for other metallic materials with suitable parameter calibration.

\section{Declaration of Competing Interest}

The authors declare that they have no known competing financial interests or personal relationships that could have appeared to influence the work reported in this paper.

\section{Acknowledgment}

This work was supported by the China Postdoctoral Science Foundation through grants No. 2019M652629 and No. 2019TQ0107. Funding from the National Natural Science Foundation of China through grant No. 52005199 is gratefully acknowledged.

\section{References}

[1] Brehl D, Dow T. Review of vibration-assisted machining. Precis Eng 2008;32(3): 153-72.

[2] Moufki A, Molinari A, Dudzinski D. Modelling of orthogonal cutting with a temperature dependent friction law. J Mech Phys Solids 1998;46(10):2103-38.

[3] Marinov VR. Hybrid analytical-numerical solution for the shear angle in orthogonal metal cutting — part I: theoretical foundation. Int J Mech Sci 2001;43 (2):399-414.

[4] Bahi S, Nouari M, Moufki A, Mansori ME, Molinari A. Hybrid modelling of sliding-sticking zones at the tool-chip interface under dry machining and tool wear analysis. Wear 2012;286:45-54.

[5] Shamoto E, Altintas Y. Prediction of shear angle in oblique cutting with maximum shear stress and minimum energy principles. ASME J Manuf Sci E 1999;121: 399-407.

[6] Olxey P. The mechanics of machining. Chichester: Ellis Horwood; 1989.

[7] Wright P. Predicting the shear plane angle in machining from workmaterial strainhardening characteristics. J Eng Ind 1982;104:285.

[8] Lee E. The theory of plasticity applied to a problem of machining. ASME J Appl Mech 1951;18:405.

[9] Merchant ME. Mechanics of the metal cutting process. I. Orthogonal cutting and a type 2 chip. J Appl Phys 1945;16(5):267-75.

[10] Childs T. Friction modelling in metal cutting. Wear 2006;260(3):310-8.

[11] Ozlu E, Budak E, Molinari A. Analytical and experimental investigation of rake contact and friction behavior in metal cutting. Int J Mach Tool Manu 2009;49(11): 865-75.

[12] Molinari A, Cheriguene R, Miguélez H. Numerical and analytical modeling of orthogonal cutting: the link between local variables and global contact characteristics. Int J Mech Sci 2011;53(3):183-206.

[13] Özel T. The influence of friction models on finite element simulations of machining. Int J Mach Tool Manu 2006;46(5):518-30.

[14] Hu Y, Shao J. The slip line cutting model of ultrasonic vibrating cutting. J Guangxi Univ (Nat Sci Edit) 1989;4:24-7.

[15] Zhang X, Arif M, Liu K, Kumar AS, Rahman M. A model to predict the critical undeformed chip thickness in vibration-assisted machining of brittle materials. Int J Mach Tool Manu 2013;69:57-66.

[16] Arefin S, Zhang X, Anantharajan SK, Liu K, Neo DWK. An analytical model for determining the shear angle in $1 \mathrm{D}$ vibration-assisted micro machining. Nanomanuf Metrol 2019;2(4):199-214.

[17] Bai W, Sun R, Gao Y, Leopold J. Analysis and modeling of force in orthogonal elliptical vibration cutting. Int J Adv Manuf Tech 2016;83(5-8):1025-36.

[18] Amini S, Kazemiyoun M. Effect of ultrasonic vibrations on chip-tool contact zone in turning of AISI304. Mater Manuf Process 2014;29(5):627-33.

[19] Jamshidi H, Nategh M. Theoretical and experimental investigation of the frictional behavior of the tool-chip interface in ultrasonic-vibration assisted turning. Int J Mach Tool Manu 2013;65:1-7.

[20] Lotfi M, Amini S. Effect of ultrasonic vibration on frictional behavior of tool-chip interface: finite element analysis and experimental study. P I Mech Eng B-J Eng 2018;232(7):1212-20.

[21] Bai W, Sun R, Roy A, Silberschmidt VV. Improved analytical prediction of chip formation in orthogonal cutting of titanium alloy Ti6Al4V. Int J Mech Sci 2017; 133:357-67.

[22] Childs TH, Maekawa K, Obikawa T, Yamane Y. Metal machining: theory and applications. Butterworth-Heinemann; 2000.

[23] Bai W, Sun R, Leopold J, Silberschmidt VV. Microstructural evolution of Ti6Al4V in ultrasonically assisted cutting: numerical modelling and experimental analysis. Ultrasonics 2017;78:70-82. 